\title{
Ophthalmic Artery Doppler Velocimetric Values in Pregnant Women at Risk for Preeclampsia
}

\author{
Denise S. Matias, MD, Rigman F. Costa, MD, Bruno Matias, MD, Léo Gordiano, Luis C. L. Correia, MD, PhD
}

Received December 20, 2011, from the Bahiana School of Medicine and Public Health, Salvador, Bahia, Brazil (D.S.M., L.C.L.C.); Institute of Perinatology of Bahia, Salvador, Bahia, Brazil (D.S.M., R.F.C.); Santo Amaro Hospital, JoséSilveira Foundation, Salvador, Bahia, Brazil (B.M.); and Federal University of Bahia, Salvador, Bahia, Brazil (L.G.). Revision requested January 7, 2012. Revised manuscript accepted for publication March 25, 2012.

Address correspondence to Luis C.L. Correia, MD, PhD, Avenida Euclides da Cunha 403/1003, 40150-120 Salvador-BA, Brazil.

E-mail:dmatias@ultradiag.com.br
Objectives - The purposes of this study were to describe ophthalmic artery Doppler parameters in pregnant women at risk for preeclampsia in the second trimester, compare them with reference values described in women with healthy pregnancies, and evaluate the association between gestational age and Doppler parameters.

Methods - We conducted an observational study with prospective data collection in which measurements of 7 ophthalmic artery Doppler parameters were obtained in 274 women at 20 to 28 weeks' gestation with clinical risk factors for preeclampsia. The following measurements were obtained: peak systolic velocity, mesodiastolic velocity, mean velocity, end-diastolic velocity, resistive index, pulsatility index, and peak ratio. The observed Doppler values were compared with reference values by a 1 -sample $t$ test. To test for linear associations between gestational age and Doppler parameters, the Pearson correlation coefficient was used.

Results - Significantly higher ophthalmic artery Doppler velocimetric parameters were observed when compared with the reference values. The mean values of these parameters \pm SD were as follows: peak systolic velocity, $37.9 \pm 10.3 \mathrm{~cm} / \mathrm{s}$ (reference, $34.2 \mathrm{~cm} / \mathrm{s}$; $P<.001$ ); end-diastolic velocity, $7.2 \pm 3.1 \mathrm{~cm} / \mathrm{s}$ (reference, $6.8 \mathrm{~cm} / \mathrm{s} ; P=.03$ ); resistive index, $0.81 \pm 0.07$ (reference, $0.70 ; P<.001$ ); pulsatility index, $2.17 \pm 0.53$ (reference, 1.80; $P<.001$ ); and peak ratio, $0.53 \pm 0.12$ (reference, $0.40 ; P<.001$ ). There was no correlation between the Doppler parameters and gestational age.

Conclusions - Ophthalmic Doppler parameters of women at risk for preeclampsia are significantly higher than reference values for healthy pregnancies, which may indicate an inadequate vascular response to persistent peripheral resistance. Gestational age during the second trimester is not associated with ophthalmic artery Doppler parameters in pregnant women at risk for preeclampsia.

Key Words - Doppler velocimetry; ophthalmic artery; preeclampsia; pregnancy

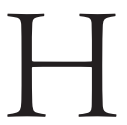
ypertensive disorders during pregnancy, especially preeclampsia, remain major causes of maternal deaths, and most of these deaths result from brain damage. These disorders affect up to $10 \%$ of all pregnancies, and it is estimated that more than $25 \%$ of women with preexisting risk factors will have preeclampsia. ${ }^{1}$

Preeclampsia is a multisystemic disorder characterized by hypertension and proteinuria, which occurs after the 20th week of pregnancy and resolves postpartum. ${ }^{2}$ In more severe cases, deterioration of renal function, hepatic and hematologic involvement, pulmonary edema, stroke, and neurologic involvement may occur. The fetus may have serious clinical complications such as restricted intrauterine growth and prematurity, which are associated with an increased 
risk of delays in neuropsychomotor development and chronic diseases in childhood. In the long run, both the woman and fetus affected by this disorder may be predisposed to cardiovascular diseases. ${ }^{3,4}$ The ability to predict this condition would be very useful because it would enable vigilance directed toward women at high risk of developing the disorder, allowing for the adoption of prophylactic and, when available, therapeutic measures. Moreover, predictive testing could facilitate research on preventive interventions, defining at-risk patients who should be included in clinical studies.

The first studies that evaluated color Doppler sonography of the orbital vessel in the United States date back to the 1980s, when Erickson et $\mathrm{al}^{5}$ published their initial experience in the evaluation of normal and abnormal orbits, and since then, this technique has been used to obtain information from the orbital vessels in a noninvasive and selective way. ${ }^{6,7}$ The ophthalmic and central retinal arteries have embryologic, anatomic, and functional similarities with small-caliber intracranial arteries. ${ }^{8}$ Therefore, the Doppler study of these vessels enables information to be gathered about the hemodynamic state of the cerebral microvasculature ${ }^{9}$ in addition to being a useful adjunct in the evaluation, treatment, and management of pregnancyinduced hypertension.

In the last few years, a number of studies have been published ${ }^{10-12}$ that reported reference values for ophthalmic artery and central retinal artery Doppler parameters throughout healthy pregnancy. This observational study included 274 pregnant women at risk for developing preeclampsia in the second half of the second trimester. The aims were to describe Doppler parameters of the ophthalmic artery, compare them with reference values reported for healthy women, and evaluate the association between gestational age and Doppler parameters.

\section{Materials and Methods}

\section{Sample Selection}

Two hundred seventy-four women between 20 and 28 weeks' gestation were included. They were patients at the high-risk prenatal clinic of the Institute of Perinatology of Bahia between March of 2010 and July of 2011, with the following risk factors for preeclampsia: primigravidas aged 18 years or younger and 40 years or older, personal or family history of preeclampsia, primipaternity or new father, twin pregnancy, obesity (body mass index $>30 \mathrm{~kg} / \mathrm{m}^{2}$ ), thrombophilia, and autoimmune diseases. Smokers as well as patients using ocular or systemic antihypertensive drugs were excluded, and each patient was studied only once. As a result of the inclusion and exclusion criteria described above, a sample population at risk for preeclampsia was selected: concerning the number of risk factors, 125 patients (45.6\%) had only $1 ; 107$ (39.1\%) had 2; 33 (12.0\%) had 3; and $9(3.3 \%)$ had 4 risk factors, the most frequent of which were early primigravida (38\%), primipaternity or new father (37.2\%), and obesity (23.4\%). Among the least frequent factors were the presence of diabetes before pregnancy (3.6\%), and late primigravida (5.8\%) (Table 1). The study was approved by the Research Ethics Committee of the School of Medicine and Public Health, Foundation for the Advancement of Science, and all patients were made aware of the purpose of the study and signed an informed consent form.

Gestational age was calculated starting from the first day of the last menstrual period for patients with reliable data and confirmed with a sonographic examination in the first trimester or early second trimester. If these data were unknown or unreliable, the gestational age was calculated by a previous sonographic examination. Complete obstetric and perinatal information was obtained from each patient.

Table 1. Demographic Characteristics and Risk Factors of the Studied Population $(n=274)$

\begin{tabular}{lc}
\hline Characteristic & Value \\
\hline Age, y & $25.5 \pm 9.4$ \\
Age range, y & $13-48$ \\
Gestational age at time of admission, wk & $23.2 \pm 1.8$ \\
Skin tone & \\
Light & $9(3.5)$ \\
Medium & $149(57.3)$ \\
Dark & $102(39.2)$ \\
No. of pregnancies & \\
1 & $139(50.7)$ \\
2 & $52(19.0)$ \\
3 & $42(15.0)$ \\
$>3$ & $41(15.0)$ \\
Parity & \\
0 & $168(61.3)$ \\
1 & $62(22.6)$ \\
2 & $24(8.8)$ \\
3 & $14(5.1)$ \\
$>3$ & $6(2.2)$ \\
Risk factors & \\
Body mass index $>30 \mathrm{~kg} / \mathrm{m}^{2}$ & $64(23.4)$ \\
Primigravida $\leq 18 \mathrm{y}$ & $105(38.3)$ \\
Primigravida $\geq 40 \mathrm{y}$ & $16(5.8)$ \\
Primipaternity & $102(37.2)$ \\
Personal history of preeclampsia & $37(13.5)$ \\
Family history of preeclampsia & $60(22.1)$ \\
Previous hypertension & $36(13.1)$ \\
Previous diabetes & $10(3.6)$ \\
Multiple gestation & $19(6.9)$ \\
\hline &
\end{tabular}

Values are mean \pm SD and number (percent) where applicable. 


\section{Study Protocol}

All ophthalmic artery Doppler velocimetric examinations were performed by a single observer (D.S.M.) at the patient's admission into the study using high-resolution equipment (SonoAce 8000 ultrasound system; Samsung Medison, Seoul, Korea) with an electronic linear transducer at the frequency of 7.5 MHz. Patients were examined in a supine position with a head tilt of approximately $15^{\circ}$ after a rest period of at least 10 minutes.

After a small amount of methylcellulose gel was applied over the closed eyelid, the transducer was positioned horizontally without applying pressure to the eye and toggled up and down until the ophthalmic artery was identified approximately $15 \mathrm{~mm}$ from the eyeball using color Doppler imaging. Five flow velocity waveforms of similar size and shape were obtained by spectral Doppler imaging; then measurements of the Doppler indices were taken from 1 waveform. The variables analyzed were the peak systolic velocity, end-diastolic velocity, peak mesodiastolic velocity, mean velocity, resistive index, pulsatility index, and peak ratio. All indices were automatically calculated by the ultrasound equipment, except the peak ratio, which was calculated by the ratio between the peak mesodiastolic velocity and peak systolic velocity. ${ }^{13}$

Only the right eye was examined because previous studies ${ }^{11,14,15}$ have shown no statistically significant differences between the blood flow in each eye. The angle of insonation between the ultrasound beam and the vessel orientation was corrected to less than $20^{\circ}$, and the gain was adjusted individually for each examination and maintained constant throughout the examination. The pulse repetition frequency of the equipment was adjusted to avoid aliasing $(5208 \mathrm{~Hz})$, and the size of the sample volume was established at $2 \mathrm{~mm}$.

\section{Data Analysis}

Initially, the Doppler parameters were expressed as mean \pm standard deviation, and for each gestational age group, $95 \%$ confidence intervals (mean $\pm 1.96 \mathrm{SDs}$ ) were reported. The gestational age groups were defined at intervals of 1 week between 20.01 and 28.00 weeks. The mean values found in our sample of patients at risk for preeclampsia were then compared with reference values obtained from a previous study ${ }^{11}$ of a Brazilian population of healthy pregnant women of similar age groups (25.5 \pm 9.4 years; reference, $26.7 \pm 4.1$ years) in the same gestational age ranges (20-28 weeks). For the comparison of these data, a 1-sample $t$ test was used. Finally, the Pearson correlation coefficient was used to test for a linear association between Doppler parameters and gestational age. An $\alpha$ error of $5 \%$ was accepted for all tests. Analyses were performed with SPSS version 17.0 software for Windows (SPSS Inc, Chicago, IL).

\section{Results}

The following mean velocities and impedance indices observed had significantly higher values than their respective reference values ${ }^{11}$ : peak systolic velocity, $37.9 \pm 10.3$ $\mathrm{cm} / \mathrm{s}$ (reference, $34.2 \mathrm{~cm} / \mathrm{s} ; P<.001$ ); end-diastolic velocity, $7.2 \pm 3.1 \mathrm{~cm} / \mathrm{s}$ (reference, $6.8 \mathrm{~cm} / \mathrm{s} ; P=.03$ ); resistive index, $0.81 \pm 0.07$ (reference, $0.70 ; P<.001$ ); pulsatility index, $2.17 \pm 0.53$ (reference, $1.80 ; P<.001$ ); and peak ratio, $0.53 \pm 0.12$ (reference, $0.40 ; P<.001$ ).

The mean and $95 \%$ confidence intervals of the Doppler parameters for each gestational age group evaluated are shown in Table 2. There was no correlation between gestational age and the Doppler parameters: peak systolic velocity $(r=0.059 ; P=0.33)$; end-diastolic velocity $(r=0.068$; $P=.26)$; peak mesodiastolic velocity $(r=0.030 ; P=.62)$;

Table 2. Ophthalmic Artery Doppler Parameters Between 20 and 28 Weeks of Pregnancy

\begin{tabular}{lccccccc}
\hline $\begin{array}{l}\text { Gestational } \\
\text { Age, } \mathbf{w k}\end{array}$ & $\mathbf{P S V}, \mathbf{c m} / \mathbf{s}$ & $\mathbf{E D V}, \mathbf{c m} / \mathbf{s}$ & $\mathbf{P M D V}, \mathbf{c m} / \mathbf{s}$ & $\mathbf{M V}, \mathbf{c m} / \mathbf{s}$ & $\mathbf{R I}$ & PI & PR \\
\hline $20.01-21.00$ & $36.9(33.7-40.1)$ & $6.0(5.0-7.0)$ & $18.8(16.3-21.4)$ & $12.7(11.2-14.3)$ & $0.83(0.81-0.86)$ & $2.53(2.16-2.90)$ & $0.52(0.45-0.58)$ \\
$21.01-22.00$ & $36.8(33.0-40.5)$ & $6.7(5.8-7.6)$ & $19.4(17.4-21.4)$ & $14.3(12.7-15.9)$ & $0.81(0.80-0.84)$ & $2.17(2.00-2.33)$ & $0.53(0.50-0.57)$ \\
$22.01-23.00$ & $37.4(35.2-39.6)$ & $7.3(6.6-8.1)$ & $20.1(18.4-21.8)$ & $14.6(13.6-15.7)$ & $0.80(0.79-0.82)$ & $2.13(2.02-2.24)$ & $0.54(0.51-0.57)$ \\
$23.01-24.00$ & $36.5(33.7-39.3)$ & $7.2(6.4-8.1)$ & $19.6(17.8-21.5)$ & $14.5(13.2-15.8)$ & $0.79(0.77-0.81)$ & $2.08(1.93-2.23)$ & $0.54(0.51-0.58)$ \\
$24.01-25.00$ & $41.9(38.0-45.8)$ & $7.9(6.6-9.2)$ & $22.2(19.5-25.0)$ & $16.5(14.6-18.4)$ & $0.81(0.80-0.83)$ & $2.14(2.00-2.28)$ & $0.53(0.50-0.57)$ \\
$25.01-26.00$ & $39.9(36.1-43.7)$ & $8.1(6.8-9.3)$ & $20.6(17.8-23.4)$ & $15.7(13.6-17.8)$ & $0.81(0.79-0.84)$ & $2.21(1.94-2.47)$ & $0.51(0.45-0.57)$ \\
$26.01-27.00$ & $35.7(29.9-41.5)$ & $5.7(4.6-6.8)$ & $17.4(14.0-20.7)$ & $12.8(10.5-15.0)$ & $0.84(0.82-0.86)$ & $2.37(2.17-2.57)$ & $0.50(0.44-0.56)$ \\
$27.01-28.00$ & $37.9(31.0-44.7)$ & $8.3(5.2-1.4)$ & $21.1(16.4-25.8)$ & $15.4(11.4-19.5)$ & $0.80(0.75-0.85)$ & $1.97(1.65-2.30)$ & $0.55(0.45-0.65)$ \\
\hline
\end{tabular}

Values are mean (95\% confidence interval). EDV indicates end-diastolic velocity; MV, mean velocity; OA, ophthalmic artery; PI, pulsatility index; PMDV, peak mesodiastolic velocity; PR, peak ratio; PSV, peak systolic velocity; and RI, resistive index. 
mean velocity $(r=0.070 ; P=.26)$; resistive index $(r=0.007$; $P=.11)$; pulsatility index $(r=-0.040 ; P=.51)$; and peak ratio $(r=-0.035 ; P=.56)$. These findings show that there were no significant variations between these indices throughout the second half of the second trimester of pregnancy (Figure 1).

\section{Discussion}

This study indicates that ophthalmic artery Doppler parameters in pregnant women at risk for preeclampsia are significantly different from the reference values that have been reported from healthy pregnancies. In addition, there was no correlation between these parameters and gestational age.

In healthy pregnancy, there is a decrease in vascular reactivity to vasoactive peptides in response to an adequate trophoblastic invasion in the maternal spiral arterioles, a phenomenon that completes itselfbetween 20 and 22 weeks of pregnancy ${ }^{16}$ and reflects an increase in the production of endothelium-derived relaxing factors, principally those that are dependent on nitric oxide. In preeclampsia, hyperreactivity to these vasoactive hormones occurs due to poor placental implantation, an abnormality that can be detected even before the hypertension becomes apparent. ${ }^{17}$ The biggest pathologic change in preeclampsia seems to be related to general arteriolar vasoconstriction, including the eye area, whose involvement has been well documented. ${ }^{1}$ This pathophysiologic process begins months before the clinical disease and can be present as early as the end of the first trimester. ${ }^{18}$

We have hypothesized that the findings in this study may have been due to an inadequate vascular response in pregnant women with risk factors for preeclampsia, with vascular resistance greater than that found in normal pregnant women, as indicated by velocities and impedance

Figure 1. Correlation between the most important ophthalmic artery Doppler velocimetric parameters and gestational age.
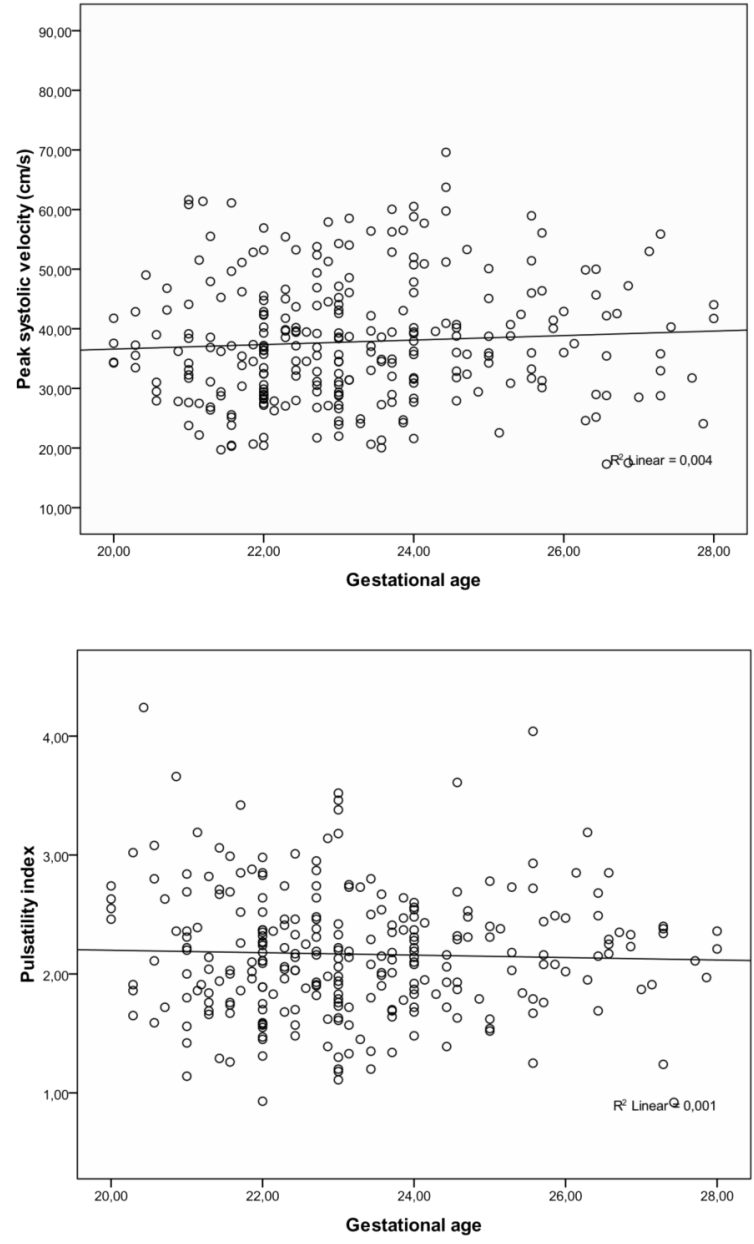
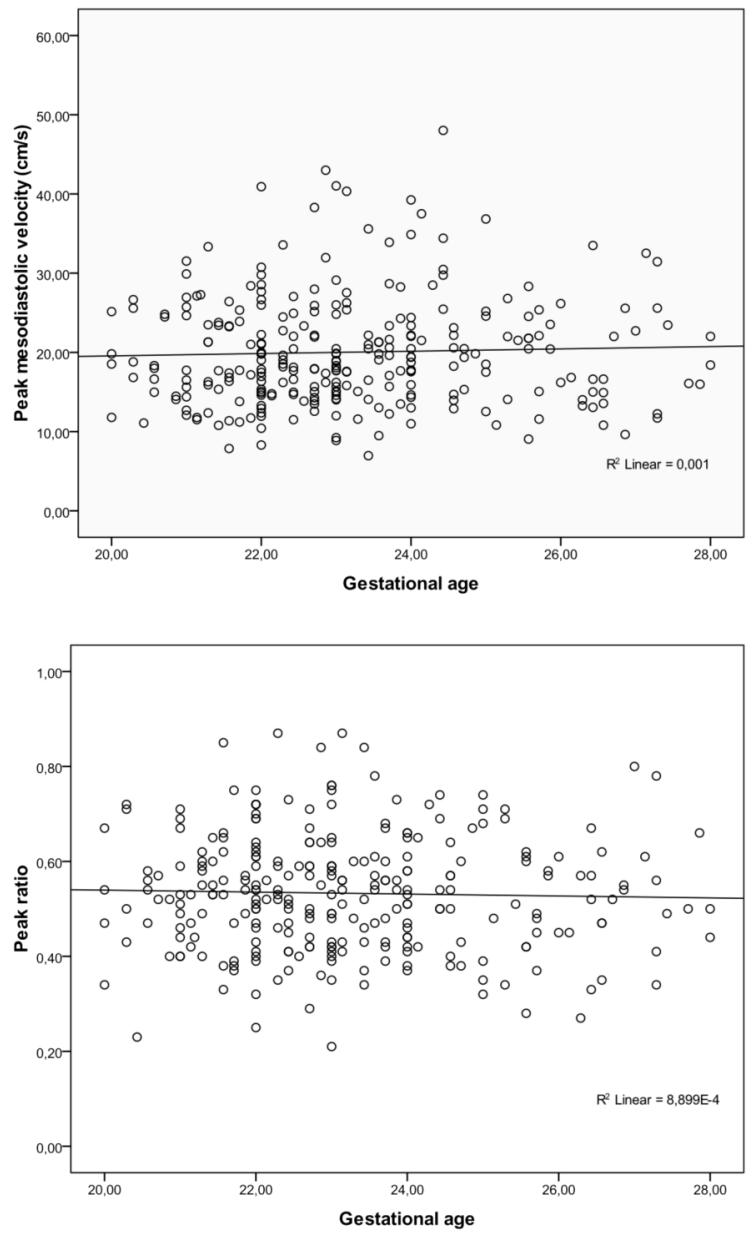
ratios higher than expected for a population of healthy pregnant women. The finding that no Doppler parameter was correlated with gestational age corroborates the idea that these women are at risk of developing preeclampsia because what is expected is an increase in the diastolic velocity and a decrease in the impedance indices along the course of pregnancy due to the vasodilatation that occurs in a healthy pregnancy. That is, the decrease in peripheral resistance compared with preexisting values would be responsible for the variation of hemodynamic parameters during pregnancy. The works of Ohno et $\mathrm{al}^{19}$ and Mackenzie et a ${ }^{12}$ reinforce this idea. Ohno et a ${ }^{19}$ evaluated 118 pregnant women without risk factors between 20 and 41 weeks' gestation and reported a negative correlation between the pulsatility index and gestational age. The same correlation was shown by Mackenzie et $\mathrm{al}^{12}$ in a study of 126 healthy pregnancies that evaluated the resistive index and systolic/diastolic ratio; these findings were attributed to an increase in diastolic flow along the course of normal pregnancy caused by a decrease in peripheral resistance. The highest velocities and impedance indices found in our study indicate vasoconstriction, similar to what is seen in the uterine arteries, where an increase in the systolic velocity and resistance indices is related to elevated peripheral resistance due to poor placental implantation.

With regard to the predictive values of Doppler velocimetry of the orbital vessels and especially the ophthalmic artery in relation to the development of preeclampsia, to our knowledge, there are no published works that evaluated their accuracy with that objective. This study has the limitation of being merely descriptive; therefore, it does not make it possible to validate the examination as a predictor, nor does it determine causation. However, it suggests a prognostic value, requiring prospective cohort studies with large samples size to test this hypothesis.

The impedance indices and velocities are important for detecting changes in blood flow, ${ }^{20}$ and in this study, these parameters were examined in an at-risk population with a substantial sample size. The early identification of women at risk for the development of preeclampsia is essential to reducing maternal and perinatal morbidity and mortality and would allow for closer monitoring and the adoption of prophylactic and therapeutic measures. The ophthalmic artery Doppler parameters obtained and described in this study are relevant and may be used as adjuncts to identify these women. As far as we know, a study in which the values of all ophthalmic artery Doppler parameters were reported in a large number of pregnant women with 1 or more risk factors for this condition has not been reported previously.
In conclusion, the ophthalmic artery flow parameters in pregnant women with risk factors for the development of preeclampsia are significantly higher than the reference values for healthy pregnancies. This finding suggests that there is potential for their use in predicting this serious complication in the pregnancy-puerperal cycle.

\section{References}

1. Wagner SJ, Barac S, Garovic VD. Hypertensive pregnancy disorders: current concepts.J Clin Hypertens (Greenwich) 2007; 9:560-566.

2. Report of the National High Blood Pressure Education Program Working Group on High Blood Pressure in Pregnancy. Am J Obstet Gynecol 2000; 183:S1-S22.

3. Dornhöfer N, Stepan H. Preeclampsia: more than a pregnancy complication. Hum Ontogenet 2008; 2:29-38.

4. Thadhani R, Solomon CG. Preeclampsia: a glimpse into the future? NEngl JMed 2008; 359:858-860.

5. Erickson SJ, Hendrix LE, Massaro BM, et al. Color Doppler flow imaging of the normal and abnormal orbit. Radiology 1989; 173:511-516.

6. Orge F, Harris A, Kagemann L, et al. The first technique for noninvasive measurement of volumetric ophthalmic artery blood flow in humans. BrJ Ophthalmol 2002; 86:1216-1219.

7. Williamson TH, Harris A. Ocular blood flow measurement. Br J Ophthalmol 1994; 78:939-945.

8. Belfort MA, Saade GR, Snabes M, et al. Gynecology: hormonal status affects the reactivity of the cerebral vasculature. Am J Obstet Gynecol 1995; 172:1273-1278.

9. Harris A, Martin B.Color Doppler imaging of the ophthalmic artery: a measure of cerebral blood flow? J Cardiothorac Vasc Anesth 1999; 13:659-660.

10. Carneiro RS, Sass N, Diniz AL, Souza EV, Torloni MR, Moron AF. Ophthalmic artery Doppler velocimetry in health pregnancy. In J Gynaecol Obstet 2008; 100:211-215.

11. Diniz AL, Moron AF, Santos MC, Pires CR. Dopplervelocimetria das artérias oftálmica e central da retina em gestantes normais. Rev Bras Ginecol Obstet 2005; 27:168-173.

12. Mackenzie F, De Vermette R, Nimrod C, Boisvert D, Jackson B. Doppler sonographic studies on the ophthalmic and central retinal arteries in the gravid woman. JUltrasound Med 1995; 14:643-647.

13. Takata M,Nakatsuka M, Kudo T. Differential blood flow in uterine, ophthalmic, and brachial arteries of preeclamptic women. Obstet Gynecol 2002; 100:931-939.

14. Hata $\mathrm{T}$, Senoh D, Hata K, Kitao M. Ophthalmic artery velocimetry in pregnant women. Lancet 1992; 340:182-183.

15. Lieb WE, Cohen SM, Merton DA, Shields JA, Mitchell DG, Goldberg BB. Color Doppler Imaging of the eye and orbit: technique and normal vascular anatomy. Arch Ophthalmol 1991; 109:527-531.

16. Cnossen JS, van der Post J, Mol BWJ, Kahn KS, Meads CA, ter Riet C. Prediction of preeclampsia: a protocol for systematic reviews of test accuracy. BMC Pregnancy Childbirth 2006; 6:1-8. 
Matias et al—Ophthalmic Artery Doppler Velocimetric Values and Preeclampsia

17. Pascoal IF. Pré-eclâmpsia: mecanismos fisiopatológicos e suas implicações terapêuticas. Rev Bras Hipertens 2000; 9:256-261.

18. Easterling TR, Benedetti TJ.Preeclampsia: a hyperdinamic disease model. AmJ Obstet Gynecol 1989; 160:1447-1453.

19. Ohno Y, Kawai M, Wakahara Y, Kitagawa T, Kakihara M, Arii Y. Ophthalmic artery velocimetry in normotensive and preeclamptic women with or without photophobia. Obstet Gynecol 1999; 94:361-363.

20. Tompson RS, Trudinger BJ, Cook CM. Doppler ultrasound waveform indices: A/B ratio, pulsatility index and Porcelot ratio. BrJObstet Gynaecol 1988; 95:581-588. 